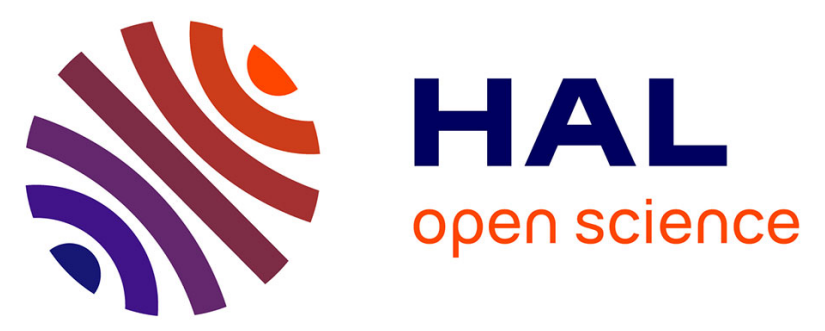

\title{
RAYONNEMENT ACOUSTIQUE D'UNE PLAQUE MINCE RECTANGULAIRE BAFFLÉE AVEC CONDITIONS LIMITES QUELCONQUES
}

\author{
A. Berry, Jean-Marie Nicolas, Jean-Louis Guyader
}

\section{- To cite this version:}

A. Berry, Jean-Marie Nicolas, Jean-Louis Guyader. RAYONNEMENT ACOUSTIQUE D'UNE PLAQUE MINCE RECTANGULAIRE BAFFLÉE AVEC CONDITIONS LIMITES QUELCONQUES. Journal de Physique Colloques, 1990, 51 (C2), pp.C2-221-C2-224. 10.1051/jphyscol:1990253 . jpa-00230674

\section{HAL Id: jpa-00230674 https://hal.science/jpa-00230674}

Submitted on 1 Jan 1990

HAL is a multi-disciplinary open access archive for the deposit and dissemination of scientific research documents, whether they are published or not. The documents may come from teaching and research institutions in France or abroad, or from public or private research centers.
L'archive ouverte pluridisciplinaire HAL, est destinée au dépôt et à la diffusion de documents scientifiques de niveau recherche, publiés ou non, émanant des établissements d'enseignement et de recherche français ou étrangers, des laboratoires publics ou privés. 
COLLOQUE DE PHYSIQUE

Colloque C2, supplément au $n^{\circ} 2$, Tome 51, Février 1990

ler Congrès Français d'Acoustique 1990

\title{
RAYONNEMENT ACOUSTIQUE D'UNE PLAQUE MINCE RECTANGULAIRE BAFFLEE AVEC CONDITIONS LIMITES QUELCONQUES
}

\author{
A. BERRY, J. NICOLÁS et J.-L. GUYADER* \\ Groupe d'Acoustique et I'Université de sherbrooke, Département de Génie \\ Mécanique, Sherbrooke, Québec J1K 2R1, Canada \\ * Laboratoire de Vibrations-Acoustique, INSA Lyon, F-69621 Villeurbanne, \\ France
}

Résumé - Une méthode de calcul analytique du rayonnement acoustique des plaques minces rectangulaires de conditions aux limites quelconques est proposée. Contrairement aux méthodes précédentes elle est générale et permet d'envisager le cas de conditions variables sur chaque bord de la plaque. La rigidité de translation des limites s'avère le paramètre dominant le rayonnement sous la fréquence critique.

Abstract - An analytical approach is developed for the acoustic radiation of thin rectangular plates with the specificity that any type of boundaries conditions can be included in the model. This make the method much more general than the previous ones. One important result is that the translation rigidity of the limits turns out to be the key parameter for the radiation under the critical frequency.

\section{INTRODUCTION}

La prédiction et le contrôle du rayonnement par les structures vibrantes est une préoccupation croissante des bureaux d'études techniques. Les structures réelles présentent rarement des conditions limites bien définies; ces conditions limites s'écartent généralement des modèles théoriques usuels, et peuvent varier d'un point à l'autre du contour. Ce papier décrit une formulation générale du rayonnement acoustique par des plaques rectangulaires dont les conditions limites sont de type élastique, et éventuellement variables le long du périmètre. L'approche suppose une densité modale de plaque faible à moyenne. La conclusion bien connue que sous la fréquence critique $[1,2]$ un panneau encastré est deux fois plus efficace en rayonnement qu'un panneau simplement supporté ne s'applique que dans le situations où la densité modale est élevée. Lorsque la densité modale est faible ou moyenne, l'approche modale est généralement utilisée [3]. Néanmoins, la complexité de la mise en oeuvre dans le cas général en limite l'emploi à des situations simples [5]. On montre ici que l'application des principes variationnels de mécanique autorise une formulation exacte dans le cas général de conditions limites élastiques en déflection et rotation. Les cas de contour appuyé, encastré, libre et guidé sont inclus dans la formulation comme cas limites. Les paramètres élastiques du contour peuvent également varier continûment le long du contour, ce qui permet d'envisager des contours vissés ou soudés par point, ou des encastrements partiels.

\section{1 - THÉORIE}

Soit une plaque mince rectangulaire (dimensions a et b), insérée dans un baffle plan, rigide, infini (Fig. 1); le système plaque-baffle sépare un milieu fluide $(Z>0)$ du vide $(Z<0)$. La charge fluide sur la plaque est négligée; l'inertie rotationnelle des sections droites et les effets de cisaillement ne sont pas pris en compte pour le mouvement de flexion. Le contour $T$ de la plaque est caractérisé en tout point $P$ par une raideur de translation $k(P)\left(\mathrm{Nm}^{-2}\right)$ et une raideur de rotation $\mathrm{C}(\mathrm{P})(\mathrm{N})$; des paramètres de contour adimensionnels sont obtenus en référant $k(P)$ et $C(P)$ à la rigidité de flexion $D$ de la plaque:

$$
k(P)=\frac{K(P) a^{3}}{D} ; \quad c(P)=\frac{C(P) a}{D}
$$

\section{A - Équation du mouvement de la plaque}

Le Hamiltonien décrivant la réponse en déplacement $w(x, y, t)$ de la plaque in-vacuo pour une excitation $f(x, y, t)$ est: 


$$
H(w)=\int_{t_{0}}^{t}\left[T\left(\frac{\partial w}{\partial t}\right)-\left(v^{\text {(plaque) }}(w)+v^{(\text {bords })}(w)\right)+w(w)\right] d t
$$

$$
V(\text { plaque })=\frac{D}{a} \int_{-1}^{+1} \int_{-1}^{+1} \frac{2}{r}\left[\left(\frac{\partial^{2} w}{\partial \alpha^{2}}\right)^{2}+r^{4}\left(\frac{\partial^{2} w}{\partial \beta^{2}}\right)^{2}+2 v r^{2} \frac{\partial^{2} w}{\partial \alpha^{2}} \frac{\partial^{2} w}{\partial \beta^{2}}+2(1-v) r^{2}\left(\frac{\partial^{2} w}{\partial \alpha \partial \beta}\right)^{2}\right] d \alpha d \beta
$$

$$
V^{(\text {bords })}=\frac{D}{a^{2}}\left\{+\int_{-1}^{+1} \frac{k}{4}\left[w^{2}(\alpha, 1)+w^{2}(\alpha,-1)\right] d \alpha+\int_{-1}^{+1} \frac{k}{4 r}\left[w^{2}(1, \beta)+w^{2}(-1, \beta)\right] d \beta\right.
$$

$$
\begin{gathered}
\left.+\int_{-1}^{+1} \operatorname{cr}^{2}\left[\left(\frac{\partial w}{\partial \beta}(\alpha, 1)\right)^{2}+\left(\frac{\partial w}{\partial \beta}(\alpha,-1)\right)^{2}\right] d \alpha+\int_{-1}^{+1} \frac{c}{r}\left[\left(\frac{\partial w}{\partial \alpha}(1, \beta)\right)^{2}+\left(\frac{\partial w}{\partial \alpha}(-1, \beta)\right)^{2}\right] d \beta\right] \\
W=\frac{a^{2}}{4 r} \int_{-1}^{+1} \int_{-1}^{+1} f(\alpha, \beta) w(\alpha, \beta) d \alpha d \beta
\end{gathered}
$$

$\alpha=\frac{2 x}{a}, \beta=\frac{2 y}{b}, r=\frac{a}{b} ;(p h)$ et $v$ sont respectivement la masse surfacique et le coefficient de Poisson de la plaque. Le principe de Hamilton permet d'identifier l'équation du mouvement de la plaque soumise aux conditions limites (1) et (2).

La minimisation de $H$ est approchée à l'aide de la méthode de Ritz: une famille de fonctions admissibles du problème $\phi_{\mathrm{n}}(\mathrm{x}) \Psi_{\mathrm{n}}(\mathrm{y})$ ayant été sélectionnée, $\mathrm{w}(\mathrm{x}, \mathrm{y}, \mathrm{t})$ est exprimé comme la combinaison:

$$
w(x, y, t)=\left\{a_{m n}(t)\right\}^{T}\left\{\phi_{m}(x) \psi_{n}(y)\right\}
$$

Les Equations de Lagrange s'appliquent à chacun des coefficients $a_{m} n$, ce qui conduit finalement au système linéaire suivant dans le cas d'une excitation sinusoïdale (pulsation $\Omega$ ):

$$
\left(-\Omega^{2}\left[\mathrm{M}_{\mathrm{mnpq}}\right]+\left[\tilde{\mathrm{K}}_{\mathrm{mnpq}}^{\text {(plaque) }}\right]+\left[\begin{array}{c}
\tilde{\mathrm{K}}_{\mathrm{mnpq}} \\
\text { (bords) }
\end{array}\right]\left\{\mathrm{a}_{\mathrm{mn}}\right\}=\left\{\mathrm{f}_{\mathrm{mn}}\right\}\right.
$$

Les coefficients de la matrice de masse $\left[\mathbf{M}_{\mathrm{mnpq}}\right]$, des matrices de raideur $\left[\mathbf{K}_{\mathrm{mnpq}}^{\text {(plaque) }}\right]$, et $\left[K_{\mathrm{mnpq}}^{(\text {bords })}\right]$ et du vecteur des forces $\left\{f_{m n}\right\}$ sont obtenus comme des produits scalaires des fonctions $\phi_{m}(x) \psi_{n}(y)$, par, respectivement un opérateur de masse, un opérateur de raideur de la plaque, un opérateur de raideur des limites, la distribution des forces appliquées. Un amortissement structural de plaque est introduit sous la forme d'une rigidité de flexion complexe:

Les fonctions $\phi_{m}(x) \psi_{n}(y)$ doivent constituer une base cinématiquement admissible du problème, c'est-à-dire doivent générer une flèche et une porte quelconque le long du contour de la plaque; la famille de monômes suivants a été retenue:

$$
\left\{\varphi_{m}(x) \Psi_{n}(y)\right\}=\left\{\left(\frac{2}{a} x\right)^{m}\left(\frac{2}{b} y\right)^{n}, m=0,1, \ldots N ; n=0,1, \ldots N\right\}
$$

\section{B) Rayonnement acoustique de la plaque}

En absence de charge fluide, le rayonnement acoustique dans le demi-espace fluide $Z>0$ est calculé à partir des vitesses vibratoires in-vacuo en appliquant l'intégrale de Rayleigh. Les indicateurs acoustiques (pression, intensité radiale, puissance) sont calculés dans le champ lointain de la plaque à partir de la transformée de Fourier 2-D des vitesses vibratoires de la plaque. La puissance rayonnée s'écrit:

$$
W=\left(\frac{a^{2}}{4 r}\right)^{2} \frac{p_{a} \Omega^{4}}{8 c \pi^{2}}\left\{a_{m n}\right\}^{T}\left[G_{m n p q}\right]\left\{a_{p q}^{*}\right\}^{T}
$$


$\rho_{a}$ et $c$ sont la densité et la célérité des ondes dans le fluide, et

$$
G_{m n q}=\int_{0}^{2 \pi} \int_{0}^{\pi / 2} \widetilde{g}_{m}\left(\lambda \frac{a}{2}\right) \tilde{g}_{m}\left(\mu \frac{b}{2}\right) \tilde{g}_{p}^{*}\left(\lambda \frac{a}{2}\right) \widetilde{g}_{q}^{*}\left(\mu \frac{b}{2}\right) \sin \theta d \theta d p
$$

avec $\lambda=\frac{\Omega}{c} \sin \theta \cos \psi ; \mu=\frac{\Omega}{c} \sin \theta \sin \psi ; g(K)=\int_{-1}^{+1} u^{P} e^{j u X} d u$.

$g_{p}(K)$ peut être calculé analytiquement. (12) est intégré numériquement. Le facteur de rayonnement pour des conditions limites données s'obtient à partir de (11) et de la vitesse quadratique de plaque.

\section{RÉSULTATS}

\section{A. Facteur de rayonnement modal pour différentes conditions limites.}

Les Fig. 2 et 3 reproduisent le facteur de rayonnement pour différents modes d'une plaque d'acier (dimensions $\mathrm{a}=455 \mathrm{~mm}, \mathrm{~b}=375 \mathrm{~mm}, \mathrm{~h}=1 \mathrm{~mm}$; fréquence critique: $12 \mathrm{kHz}$ ), dans les cas encastré, guidé et libre. La Fig. 2 montre que les panneaux appuyés et encastrés présentent sensiblement la même efficacité de rayonnement; le facteur de rayonnement est supérieur en basses fréquences dans le cas appuyé par les modes (1.1), (2.1), (2.2), et dans le cas encastré pour le mode (3.1) (cette dernière tendance se confirme pour les modes d'ordre élevé). Les conclusions de Maidanik [1] ne s'appliquent donc pas aux modes d'ordre faible. Les plaques guidée et libre admettent des modes rigides. Le mode piston (courbe 0) et la rotation rigide autour de l'axe $y$, dans le cas libre uniquement (courbe 2), sont représentés à la Fig. 3. Ces modes rigides sont les plus efficaces en rayonnement. Les modes élastiques, au contraire, présentent un faible facteur de rayonnement; ceci s'explique physiquement par les effets de court-circuit acoustique.

\section{B. Rayonnement de puissance acoustique pour différentes conditions limites.}

La Fig. 4 présente la puissance acoustique rayonnée par la plaque excitée ponctuellement en son centre, pour les quatre conditions limites décrites plus haut. L'émergence des modes résonants antisymétriques dans le spectre de puissance est évidente dans les cas appuyé et encastré, et nulle dans les cas libre et guidé, par lesquels le rayonnement des modes élastiques est masqué par celui du mouvement piston [6]. La Fig. 5 envisage une perturbation autour d'une condition limite complètement libre: des encastrements localisés sont introduits, soit au centre de chaque côté, soit à chaque coin de la plaque. Dans les deux cas, les déplacements imposés, bien que localisées, suffisent à compromettre totalement le faible rayonnement de la plaque libre.

\section{CONCLUSIONS}

L'application des principes variationnels fournit une alternative intéressante aux méthodes modales classiques pour le rayonnement acoustique de plaques rectangulaires présentant des conditions limites de type élastique en déflection-rotation. Les cas de plaques encastrees, et libres, non traités dans la littérature, ont pu être simulés. En dessous de la fréquence critique il apparaît que le rayonnement est très sensible à la raideur de translation des limites. Un montage souple en déflection le long du périmètre (cas libre et guidé) fait chuter de manière spectaculaire l'efficacité de rayonnement des modes élastiques. La présence d'encastrements partiels sur une plaque libre rehausse considérablement le rayonnement sonore de la plaque.

\section{RÉFÉRENCES}

1. G. Maidanik, "Response of ribbed panels to reverberant acoustic fields," J. Acoust. Soc. Am. 34 . 809-826 (1962)

2. A.S. Nikiforov, "Radiation of sound from a plate of finite dimensions with arbitrary boundary conditions," J. Sov. Physics Acoust. 10, 178-182 (1964)

3. N.S. Lomas, S.I. Hayek, "Vibration and acoustic radiation of elastically supported rectangular plates," J. Sound. Vib. $\underline{52}$, 1-25 (1977)

4. M.C. Gomperts, "Radiation from rigid, baffled rectangular plates with general boundary conditions," Acustica 30, 320-327 (1974)

5. M.C. Gomperts, "Sound radiation from baffled, thin, rectangular plates," Acoustica 37, (1977)

6. A. Berry, J.L. Guyader, J. Nicolas, "A general formulation for the sound radiation from rectangular, baffled plates with arbitrary boundary conditions", submitted for publication in J. Acoust. Soc. Am. (1989). 


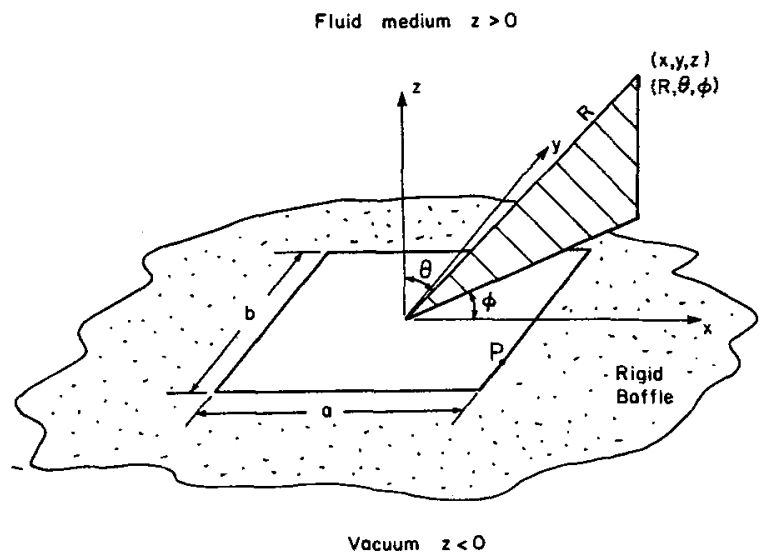

Fig. 1 - Géométrie du problème et système de coordonnées

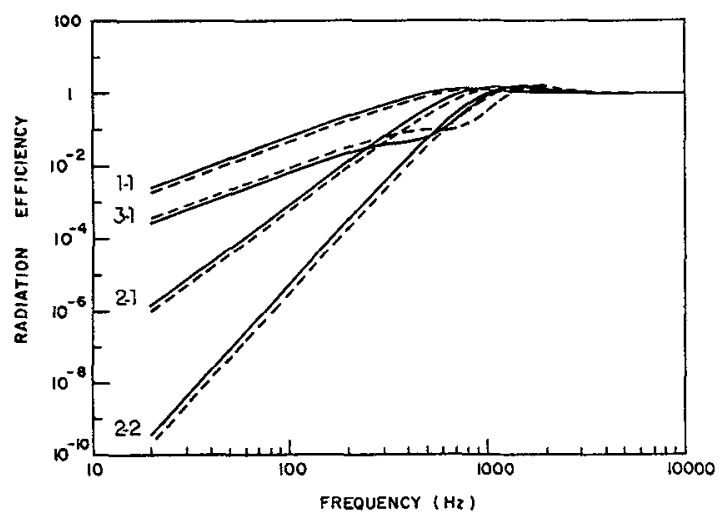

Fig. 2 - Facteur de rayonnement modal d'une plaque appuyée (trait plein), et d'une plaque encastrée (traịt discontịnu).

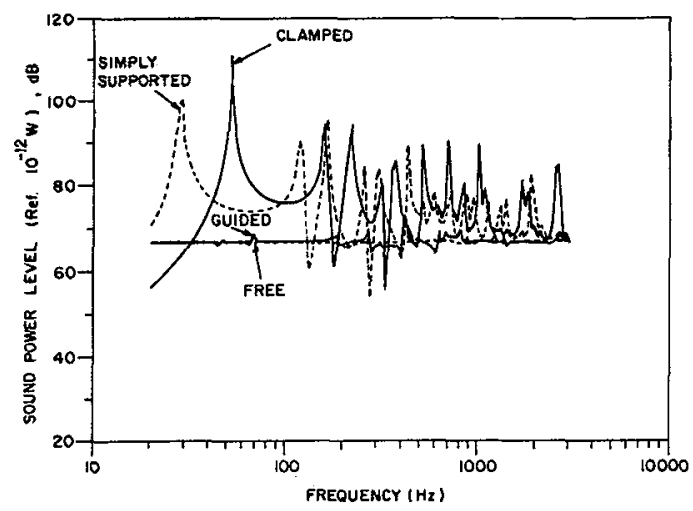

Fig. 4 - Puissance rayonnée pour différentes conditions limites. Excitation par une force ponctuelle unitaire au centre de la plaque.

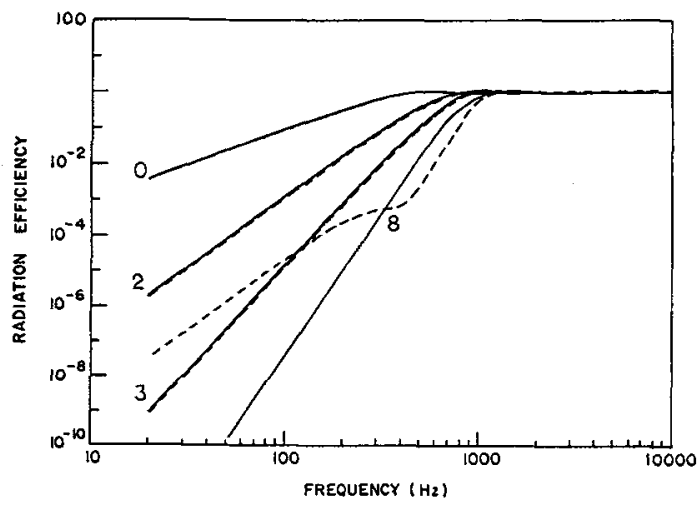

Fig. 3 - Facteur de rayonnement modal d'une plaque libre (trait plein), et d'une plaque guidêe (traịt discontinu).

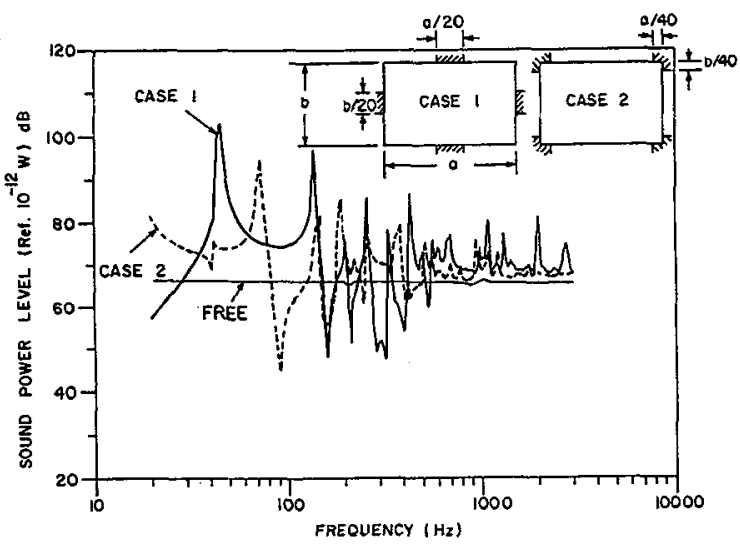

Fig. 5 - Puissance rayonnée pour des cond1tions limites partiellement libre-partiellement encastrêe. Excitation par une force ponctuelle unitalre an centre de 1 a $n 7$ arue. 